\title{
ASPECTOS ERGONÔMICOS NA TRANSFERÊNCIA DE PACIENTES: UM ESTUDO REALIZADO COM TRABALHADORES DE UMA CENTRAL DE TRANSPORTES DE UM HOSPITAL UNIVERSITÁRIO *
}

\author{
THE TRANSFER OF PATIENTS AND ITS ERGONOMIC ASPECTS: \\ A STUDY CONDUCTED AT A UNIVERSITY HOSPITAL LIFTING CENTER
}

\section{ASPECTOS ERGONÓMICOS EN LA TRANSFERENCIA DE ENFERMOS: UN ESTUDIO HECHO COM TRABAJADORES DE UNA CENTRAL DE TRANSPORTES DE UN HOSPITAL UNIVERSITÁRIO}

Cristiane Gonzales Rossi**

Rossi CG, Rocha RM, Alexandre NMC. Aspectos ergonômicos na transferência de pacientes: um estudo realizado com trabalhadores de uma central de um hospital universitário. Rev Esc Enferm USP 2001; 35(3):249-56.

\section{RESUMO}

Com o objetivo de avaliar aspectos ergonômicos na transferência de pacientes com a utilização de maca e cadeira de rodas, observou-se 249 transferências realizadas por trabalhadores da Seção de Escolta de um Hospital Universitário. Os resultados sugerem que a existência dessa equipe é uma iniciativa que deve ser estimulada mas que esses trabalhadores precisam de treinamento específico sobre movimentação e transporte de pacientes e de terem à disposição materiais auxiliares e equipamentos mecânicos.

PALAVRAS-CHAVE: Ergonomia. Transferência de pacientes. Postura.

\begin{abstract}
The purpose of this study was to evaluate ergonomic aspects involved in using a stretcher and a wheel chair to transfer patients. 249 transfers carried out by the workers of a university hospital lifting sector were observed. The observations showed that the idea of having a team to transfer patients has to be stimulated but the members of the team require specific training in handling and transferring patients. Mechanical equipment and other devices should also be available.

KEYWORDS: Ergonomics. Patient transfer. Posture.

\section{RESUMEN}

Com el objetivo de avaliar aspectos ergonómicos de transferencia de enfermos com utilización de maca y silla de ruedas, observarán se 249 transferencias realizadas por trabajadores de la Sección de Escolta (Acompañamiento) de un Hospital Universitário. Los resultados sugerem que la existencia desse equipo es una iniciativa que debrá ser estimulada. Pero esos trabajadores necessitan entrenamiento especifico sobre mobilizacion y transporte de enfermos e necessitam tener a disposicion materiales auxiliares y equipamentos mecânicos.
\end{abstract}

PALABRAS -CLAVE: Ergonomia. Transferencia de enfermos. Postura.

\footnotetext{
* Trabalho apresentado no 50 Congresso Brasileiro de Enfermagem, Salvador, BA, setembro/1998.

** $\quad$ Enfermeira de Unidade Básica de Saúde da Prefeitura Municipal de Campinas-SP E-mail: cristianegonzales@zipmail.com.br

*** Enfermeira do Hospital Municipal Walter Ferrari, Jaguariúna-SP E-mail: guilherme@galmaq.com.br Prof. Assist. Dr. do Depto. de Enfermagem da Faculdade de Ciências Médicas da UNICAMP. E-mail: neusalex@fcm.unicamp.br
} 


\section{INTRODUÇÃO}

\subsection{Considerações iniciais}

Pesquisas internacionais e nacionais têm demonstrado que os trabalhadores da área de saúde apresentam uma taxa significativamente elevada de problemas nas costas (1-6). Stubbs, Buckle, Hudson, Rivers e Worringham (4) ao realizarem uma pesquisa com enfermeiras inglesas estimaram que 750.000 dias de trabalho são perdidos anualmente devido as algias vertebrais e que uma em cada seis enfermeiras atribui este início de dor a um incidente ao movimentar pacientes.

Dessa forma, estudos têm demonstrado uma associação entre os problemas na coluna vertebral com a movimentação de pacientes acamados e com o excesso de esforço físico ao transportá-los $(2,7,8,6,6,30)$ Este fato estimulou o desenvolvimento de pesquisas sobre as técnicas de movimentação e transporte de pacientes. Verificou-se, então, que durante a realização desses procedimentos os pesos levantados por trabalhadores igualam-se ou mesmo excedem as recomendações. Além disso, são realizados sob condições desfavoráveis com número reduzido de pessoas e com equipamentos inadequados e sem manutenção (11-16').

Devido a complexidade dos fatores relacionados com as atividades que envolvem o transporte de pacientes, cada vez mais buscam-se novas abordagens para entender e reduzir os aspectos prejudiciais. A estratégia mais utilizada tem sido a administração de cursos de treinamento sobre métodos corretos de manuseio e levantamento de cargas $(17,18)$. Stubbs, Buckle, Hudson e Rivers (5) questionam essa ênfase no treinamento e ponderam que, se o trabalho é inseguro intrinsecamente, então não é somente o treinamento que corrigirá a situação e defendem uma abordagem que requer o estabelecimento de sistemas seguros de trabalho.

Pesquisadores começam a voltar seu interesse para estudos de estratégias sistematizadas dentro de uma estrutura ergonômica, o que abrange a interação entre os equipamentos, as atividades, o ambiente e o próprio pessoal $(13,14,19,20,21)$ A Organização Mundial de Saúde (22) também defende que a prevenção de lesões no sistema músculo-esquelético deve ser realizada mediante o melhoramento do ambiente, instrumentos, equipamentos e métodos de trabalho. Esta organização ressalta ainda que a prevenção destas lesões constitui o maior desafio para a ergonomia.

Considerando-se que os procedimentos de movimentar e levantar pacientes são comuns em ambiente hospitalar e que os riscos ocupacionais associados com estas técnicas são elevados, sentiu-se necessidade de realizar esse estudo. Espera-se também coletar subsídios que auxiliem no planejamento de programas de prevenção e incentivem o desenvolvimento de uma consciência crítica em relação aos efeitos do ambiente de trabalho sobre a saúde dos trabalhadores de hospital.

\subsection{Objetivo}

O presente estudo tem por objetivo avaliar aspectos ergonômicos na transferência de pacientes, utilizando maca e cadeira de rodas, por trabalhadores da Seção de Escolta de um Hospital Universitário.

\section{METODOLOGIA}

\subsection{Local}

A instituição estudada para a realização deste trabalho foi um Hospital Universitário localizado na cidade de Campinas, Estado de São Paulo, com 403 leitos.

Esse hospital possui uma Seção de Escolta à Pacientes que é um serviço organizado e chefiado por uma enfermeira. Esta seção é composta basicamente por maqueiros e, tem como objetivo ter no hospital um grupo centralizado responsável pelo transporte de pacientes e determinados materiais de laboratório. Esta equipe realiza qualquer tipo de transferência dentro do hospital e trabalha somente nos dias úteis, nos períodos da manhã (7-14 horas) e da tarde (13-20 horas).

\subsection{Instrumento de coleta de dados}

Optou-se pela observação, sendo que a análise foi feita através de uma ficha com roteiro prefixado. Este instrumento é constituído de questões fechadas e abertas e tem três partes: dados gerais das transferências, dados específicos e dados sobre o paciente. O registro dos dados foi feito pelas próprias autoras, durante a etapa de coleta de dados e o processamento dos mesmos foi realizado. manualmente.

\subsection{Amostra}

Foi realizado um plano piloto em um dia típico de trabalho junto à Seção de Escolta, quando observou-se 81 transferências de pacientes, nos turnos manhã e tarde. Após a aplicação deste, foi possivel revisar e modificar a ficha de observação e planejar o cálculo amostral.

Foram observadas 249 transferências de pacientes durante uma semana de atividades típicas da Seção de Escolta. Essa amostra aleatória simples 
foi estratificada proporcionalmente pelos turnos da manhã e da tarde. O nível de significância usado foi a $=5 \%$ e $\mathrm{d}=$ erro experimental $(5 \%)$. E importante lembrar aqui que estes valores referentes ao número de transferências a serem observadas, bem como sua distribuição quanto ao equipamento e turno foram designados pelo Setor de Estatística da Faculdade de Ciências Médicas de acordo com os dados fornecidos pelo plano piloto e pela Seção de Escolta sobre o número de transferências realizadas diária e mensalmente. Isto foi feito para que se conseguisse garantir uma amostragem significativa e real do trabalho no setor estudado.

\section{RESULTADOS E DISCUSSÃO}

\section{A - Dados gerais}

Foram observadas 249 transferências de pacientes utilizando maca e cadeira de rodas, durante uma semana típica de trabalho na Seção de Escolta do Hospital Universitário.

Tabela 1- Distribuição de transferências de pacientes realizadas pela Seção de Escolta, segundo turno e equipamento utilizado. Campinas, 1997.

\begin{tabular}{lcccccc}
\hline \multirow{2}{*}{$\begin{array}{l}\text { Equipamento } \\
\text { utilizado }\end{array}$} & \multicolumn{2}{c}{ M a n hã } & \multicolumn{2}{c}{ T a r d e } & \multicolumn{2}{c}{ T O T A L } \\
\cline { 2 - 7 } & $\mathrm{N}^{\circ}$ & \multicolumn{1}{c}{$\%$} & $\mathrm{~N}^{\circ}$ & $\%$ & $\mathrm{~N}^{\circ}$ & $\%$ \\
\hline Cadeira de rodas & 32 & 12,9 & 75 & 30,1 & 107 & 43 \\
Maca & 50 & 20,1 & 92 & 36,9 & 142 & 57 \\
\hline \multirow{2}{*}{ TOTAL } & 82 & 33,0 & 16 & 67,0 & 249 & 10 \\
\hline
\end{tabular}

Observou-se a maior parte das transferências no período da tarde, por ser neste turno de trabalho que acontecem a maior parte dos exames complementares e retornos do Centro Cirúrgico.

Para complementar estes dados, verificou-se os motivos das transferências. Os encaminhamentos e retornos de exames complementares representaram $57,8 \%$ do total. Este dado pode ser justificado devido ao hospital em estudo ser universitário e de nível terciário que acarreta no pedido de grande quantidade dos mais variados exames, tanto pela necessidade de aprendizado dos alunos como para definição e comprovação de diagnósticos.

A ida e o retorno ao Centro Cirúrgico foi outro item que apareceu freqüentemente como causa das transferências $(28,5 \%)$ e também pode ser explicado pelas características do serviço em questão, fato que faz com que este hospital receba grande número de pacientes que necessitam de maiores cuidados, entre eles, cirurgias das mais diversas. Em terceiro lugar ficou a transferência de pacientes de unidades de internação $(45,2 \%)$.

\section{B - Dados Especificos}

Para se demonstrar os tipos de transferências realizadas construiu-se a Tabela 2 .

Tabela 2 - Distribuição dos tipos de transferências de pacientes realizadas pela Seção de Escolta. Campinas, 1997.

\begin{tabular}{lcc}
\hline Tipo de transferência & $\mathrm{N}^{\circ}$ & $\%$ \\
\hline Da cama para cadeira de rodas e vice-versa & 96 & 38,6 \\
Da cama para maca e vice-versa & 92 & 36,9 \\
Da maca para maca & 38 & 15,3 \\
Da maca para maca de ambulância e & 11 & 4,4 \\
vice-versa & 5 & 2,0 \\
Da cadeira para cadeira de rodas & 5 & 2,0 \\
Da cadeira de rodas para maca de & 1 & 0,4 \\
ambulância & 1 & 0,4
\end{tabular}

Da cadeira para maca

Da maca para cadeira de rodas e vice-versa

TOTAL

249100,0

Nota-se que os tipos de transferências mais freqüentes foram da cama para cadeira de rodas ou vice-versa $(38,6 \%)$ e da cama para maca ou vice-versa $(36,9 \%)$, pois a maioria das transferências realizadas ocorrem nas unidades de internação, quando os trabalhadores da Escolta vão levar ou buscar os pacientes para exames complementares e centro cirúrgico.

Ao realizar um estudo sobre as atividades relacionadas com a movimentação de pacientes, Tuffnell (15) encontrou que os trabalhadores de um hospital movimentaram ou sentaram o paciente em primeiro lugar, sendo seguido pela transferência do paciente de cadeira para cama. Dessa forma, deve-se urgentemente buscar alternativas práticas para facilitar os procedimentos relacionados com a transferência de paciente da cama para cadeiras no meio hospitalar. As transferências de maca para maca também observadas em número relativamente alto $(15,3 \%)$, ocorreram em sua grande maioria na transferência de pacientes para o centro cirúrgico.

Tabela 3 - Distribuição das transferências de pacientes realizadas pela Seção de Escolta, de acordo com a técnica utilizada. Campinas, 1997.

\begin{tabular}{lrr} 
Técnica & $\mathrm{N}^{\circ}$ & $\%$ \\
\hline Paciente deslocou-se sozinho (sem ajuda) & 115 & 46,2 \\
Paciente foi deslocado com o auxilio de & 71 & 28,5 \\
Paciente foi deslocado com o refil de centro & 34 & 13,6 \\
Paciente deslocou-se com o auxilio da & 17 & 6,9 \\
Paciente foi sustentado nos braços & 12 & 4,8 \\
\hline TOTAL & 249 & 100,0
\end{tabular}


Verifica-se pelos dados que a equipe de Escolta permite que o paciente se desloque sozinho quando tem condições para isto. Isto ocorre quando os pacientes são independentes e constituíram 115 $(46,2 \%)$ das observações. Segundo Stubbs, Buckle, Hudson e Rivers(5) a maioria dos pacientes pode participar e cooperar nas transferências, sendo que isto contribui para sua reabilitação e seu conforto, além de reduzir o esforço da equipe.

Durante o preparo de um paciente para procedimentos que envolvem sua movimentação e transporte, deve-se orientar como se pretende movêlo e como ele pode cooperar sendo que deve também ser incentivado a ajudar sempre que possivel (23).

Por sua vez, quando o paciente não pode auxiliar, a técnica mais comum é a que utiliza um lençol e foi observada em 71 transferências $(28,5 \%)$.Com relação a isso, Narrow e Buschle (24) referem que esta é a técnica mais perigosa para a segurança do trabalhador e também descrevem medidas de prevenção para se evitar lesões nestes casos.

Outra técnica vista em 34 transferências (13,6\%) foi a utilização do refil de centro cirúrgico, que consiste em um instrumento que se acopla na maca, facilitando a entrada do paciente no centro cirúrgico. Durante este tipo de transferência ocorreram posturas inadequadas. Nota-se também que a sustentação do paciente nos braços foi menos freqüente, porém é a técnica que exige grande esforço fisico e causa posturas bastante prejudiciais à coluna vertebral.

Averiguou-se também o número total de funcionários que participaram das transferências. De uma forma geral, incluindo-se os trabalhadores da Seção de Escolta e das unidades de internação, a média de pessoas que realizaram as transferências foi de 2,3. Um estudo sobre esta questão demonstrou que geralmente de duas a três pessoas participam das transferências ${ }^{(11)}$

Percebeu-se também que em praticamente metade das transferências $(49,0 \%)$ não havia auxilio ou participação dos trabalhadores das unidades de internação onde estavam os pacientes a serem transportados. Quando existia auxílio, geralmente ocorria porque o paciente era obeso ou dependente e o pedido de ajuda partia dos próprios trabalhadores da Escolta. Em poucos casos os trabalhadores das unidades se propuseram a ajudar sem terem sido solicitados. Verificou-se ainda que nos casos onde existia auxílio, na maior parte das vezes este era realizado por um ou dois trabalhadores $(44,9 \%)$. Raros foram os casos em que três ou quatro trabalhadores auxiliaram na transferência de pacientes $(6,1 \%)$.

Nota-se que ainda existe muita resistência do pessoal das unidades de internação para auxiliar prontamente a equipe da Seção de Escolta, fazendo com que muitas vezes estes trabalhadores se sobrecarreguem, tendo que levantar cargas com pesos muito superiores aos pesos máximos indicados. Seria necessário um trabalho de conscientização com toda a equipe do Hospital Universitário, especialmente com os trabalhadores das unidades de internação, sobre a importância da colaboração principalmente em casos de pacientes graves, obesos ou dependentes (que não podem auxiliar na transferência).

Uma outra questão procurou verificar se a transferência de pacientes foi um procedimento planejado pelos trabalhadores da Seção de Escolta, sendo que em 229 transferências observadas (92\%) houve planejamento do procedimento por quem o executou. Recorda-se aqui que muitas vezes o paciente tinha condições de deslocar-se sozinho o que não exigiu um planejamento por parte da equipe.

Como falta de planejamento (8\%), observou-se mais freqüentemente que não foi dado sinal para o início da transferência, o que pode repercutir em um início não sincrônico do procedimento, somando-se ainda o fato de que em algumas transferências não foi determinado um líder, que comandasse esse início. Acredita-se que tanto a falta de liderança como o início não sincrônico podem ter culminado em um movimento descoordenado, que foi percebido em cinco transferências. A falta de planejamento foi também notada pela não retirada dos pedais da cadeira de rodas, dificultando a realização do procedimento, principalmente quando o paciente era parcialmente ou totalmente dependente, uma vez que durante a transferência o trabalhador tinha que se preocupar com a segurança do paciente e com a retirada dos pedais, adotando muitas vezes posturas incorretas. Outra condição verificada foi a não providência do suporte de soro que levava a duas situações diferentes: ou o funcionário da Seção de Escolta segurava o frasco de soro e esperava que alguém fornecesse o suporte para ele, atrasando assim o transporte, ou segurava o frasco de soro elevado durante todo o trajeto, conduzindo simultaneamente a maca ou a cadeira de rodas, com risco de lesões musculares.

As técnicas que envolvem a movimentação e o manuseio de pacientes são consideradas as mais penosas para os trabalhadores de hospital e envolvem um rigoroso planejamento para torná-las menos prejudiciais. Este planejamento deve abordar a avaliação das condições do paciente e o preparo do ambiente e equipamento, sendo um tópico importantíssimo de cursos de treinamento (23.25)

A seguir, observou-se se o espaço físico era suficiente para não limitar os movimentos da equipe ao realizar o procedimento. Notou-se que na maioria das transferências $(88,2 \%)$ o espaço fisico foi suficiente e que em 10,8\%, o espaço físico limitou os movimentos de quem as estava realizando. 
Verificou-se que os trabalhadores da Seção de Escolta algumas vezes optaram por realizar a transferência no esp'aço compreendido entre o leito do paciente e a parede, situação já descrita por Alexandre e Angerami (11) e na qual o espaço nunca era suficiente. E importante salientar que neste espaço há atualmente uma pia e que normalmente a mesa de cabeceira também se situa aí, limitando ainda mais o espaço para a transferência e obrigando o deslocamento do leito do paciente para se colocar a maca ao lado da cama. Algumas vezes o trabalhador ficava prensado entre a maca e a pia, dificultando a sua movimentação durante o procedimento.

Um dos princípios básicos da movimentação e transferência de pacientes é que se faça primeiramente a verificação do local, com posterior remoção de todos os obstáculos. Somente depois dessas providências deve-se executar esses procedimentos (26).

Alguns autores apontam que o espaço fisico restrito ao limitar os movimentos é um dos fatores causadores de dores nas costas no pessoal que realiza o transporte ou a transferência de pacientes, sendo que alguns deles descrevem condições que colaboram para a manutenção deste espaço insuficiente como: a disposição das camas no quarto, com a inexistência de distâncias adequadas entre os leitos, excesso de mobiliário e grande quantidade de objetos $(3,11)$

Quanto a relação entre as alturas de maca e cama, notou-se através dos dados coletados que em $64,1 \%$ das observações a maca era mais alta que a cama, dificultando a transposição do paciente, sobretudo da cama para a maca e exigindo um esforço adicional por parte dos trabalhadores do setor. Isso acontecia principalmente quando se tratava da transferência de pacientes dependentes e fazia com que os trabalhadores tivessem que levantar o paciente com o lençol, sendo que o desejável seria que o deslocassem, puxando o lençol. A utilização da prática de levantar o paciente com o lençol pode estar acarretando sérios danos a coluna vertebral e a estruturas osteomusculares nestes trabalhadores. Em $33,7 \%$ dos casos maca e cama apresentavam a mesma altura e em $2,2 \%$ a cama era mais alta.

A diferença nas alturas da maca e da cama também é um fator descrito como causador de dores nas costas por vários autores, que enfatizam ainda a necessidade de se adquirir camas e macas de alturas ajustáveis ou de se padronizar suas alturas em um mesmo nível $(1,3,11,27)$

Verificou-se em seguida, se os equipamentos utilizados para a transferência dos pacientes (maca, cadeira de rodas e refil de maca de centro cirúrgico) funcionavam adequadamente. Em 45,7\% dos casos notaram-se problemas relacionados aos equipamentos, que são mostrados na tabela 4.
Tabela 4 - Relação de problemas observados nos equipamentos utilizados para a transferência de pacientes pela Seção de Escolta. Campinas, 1997.

\begin{tabular}{ll}
\hline Problemas observados nos equipamentos & $\mathrm{N}^{\circ}$ \\
\hline Cadeira e maca sem travas & 103 \\
$\begin{array}{l}\text { Cadeira, maca e refil de maca de centro } \\
\text { cirúrgico }\end{array}$ & 20 \\
$\begin{array}{l}\text { com rodas de difícil movimentação } \\
\text { Cadeira de rodas sem pedal }\end{array}$ & 12 \\
$\begin{array}{l}\text { Cadeira de rodas sem local para colocação } \\
\text { de suporte de soro }\end{array}$ & 2 \\
\hline TOTAL & 147 \\
\hline
\end{tabular}

O principal problema encontrado foi a ausência de travas em macas e em cadeiras de rodas, exigindo que uma pessoa se encarregue de apoiar o equipamento para que ele não deslize, comprometendo a segurança do paciente. Enfatiza-se aqui que há uma padronização na Seção de Escolta segundo a qual a transferência em cadeira de rodas é realizada por um trabalhador e a transferência em maca por dois trabalhadores do setor, que acabam assumindo posturas incorretas para segurar a cadeira ou maca e auxiliar o paciente ao mesmo tempo.

A existência de cadeiras de rodas, macas e refis de macas com rodas de dificil movimentação foi também um problema notado e que compromete a condução desses equipamentos pelos corredores do hospital, exigindo o emprego de um esforço físico maior por parte dos trabalhadores do setor.

Outros defeitos observados foram: a falta dos pedais em cadeiras de rodas, que interfere na segurança e no conforto do paciente a ser transportado e a ausência de local para colocação do suporte de soro, fazendo com que o trabalhador da Seção de Escolta tenha que improvisar, fixando-o com esparadrapo, por exemplo, ou segurando o frasco de soro durante todo o trajeto. Esse último problema foi percebido principalmente no turno da tarde, no qual o volume de trabalho era maior, impedindo uma seleção do equipamento, que implicaria em uma demora ainda maior no atendimento às solicitações.

Alexandre e Angerami (11) apontam que o controle do ambiente e dos equipamentos é fundamental para o bom andamento do trabalho e para a prevenção de doenças profissionais e acidentes de trabalho. As mesmas autoras colocam que a falta de manutenção de equipamentos é um dos fatores que torna o trabalho mais árduo para os trabalhadores de hospital.

Na questão seguinte, procurou-se verificar a presença de problemas com o piso, que pudessem de alguma forma interferir no trabalho realizado pelos trabalhadores da Seção de Escolta. Na maioria das transferências $(98,4 \%)$ não foram detectados problemas com o piso e nos casos em que estavam presentes $(1,6 \%)$, eram referentes ao fato do piso estar sendo lavado, o que o tornava escorregadio, 
aumentando a possibilidade de ocorrência de quedas. Uma abordagem ergonômica do manuseio de cargas envolve a avaliação de todos os riscos, incluindo o piso que é considerado uma das causas de lesões dorsais quando estão irregulares ou escorregadios $(26,28)$. A utilização de sapatos com solas apropriadas ajudaria a resolver esse problema (29)

Não se verificou o uso de equipamentos auxiliares ou mecânicos em nenhuma transferência realizada pelos trabalhadores da Seção de Escolta. A falta de equipamentos auxiliares para movimentação e transporte de pacientes também tem sido apontado como fator causal de lesões dorsais em trabalhadores de hospital (39-33). De acordo com McAtamney e Corlett (20), deve-se evitar levantamentos e transferências manuais sempre que possivel, utilizando-se ao máximo materiais e equipamentos auxiliares.

Marziale (3) coloca a necessidade de se estar adequando as condições de trabalho às características da população trabalhadora e cita como uma das medidas necessárias a aquisição de equipamentos auxiliares como o elevador de paciente para facilitar o transporte de pacientes da cama para maca ou cadeira. Alexandre, Angerami e Moreira Filho(1) citam que já existem no comércio equipamentos brasileiros que auxiliam na movimentação e levantamento de pacientes e que a não utilização destes no ambiente hospitalar brasileiro merece um estudo específico.

Em seguida, levantou-se outros fatores que prejudicaram a transferência de pacientes e que foram percebidos em $12,8 \%$ das observações. Esses fatores dividiram-se em relacionados ao paciente e a equipe. Notou-se que dentro das categorias apontadas, os fatores que apareceram com uma freqüência maior foram os relacionados ao paciente, como as dificuldades em se transferir o paciente que está em isolamento, que se referem ao complicado acesso a esse paciente, sendo que neste caso, geralmente o procedimento é realizado por um trabalhador da Seção de Escolta e por um funcionário da unidade de internação correspondente, sendo o trabalho bastante penoso no caso do paciente ser dependente.

Pacientes obesos, que não colaboram e com aparelhos e sondas também são apontados por Alexandre e Angerami(11), pois exigem um esforço maior por parte de quem está fazendo a transferência, sobretudo se estiverem somados a um número reduzido de pessoas para executá-la. Quando há excesso de aparelhos e sondas, muitas vezes se torna necessário uma pessoa a mais para auxiliar na hora da transferência e no transporte, já que tanto as macas como as cadeiras de rodas não possuem recipientes ou locais próprios para alojá-los. Um número insuficiente de pessoas e a falta de auxílio foram também observados por Alexandre e Angerami(11) e demonstram a necessidade de haver uma maior colaboração dos profissionais das unidades de internação com os trabalhadores da Seção de Escolta.

Em seguida, procurou-se observar a presença de posturas incorretas nos trabalhadores da Seção de Escolta no momento da transferência de pacientes ( tabela 5).

Tabela 5 - Posturas incorretas observadas nos trabalhadores da Seção de Escolta ao transferir pacientes. Campinas, 1997.

\begin{tabular}{lc}
\hline Posturas incorretas & $\mathrm{N}^{\circ}$ \\
\hline Inclinação das costas & 75 \\
Torção do tronco & 47 \\
Não flexão dos joelhos & 36 \\
Posicionamento incorreto dos pés & 33 \\
\hline TOTAL & 191 \\
\hline
\end{tabular}

Para realizar a transferência de um paciente de forma correta é necessário que se conheça e aplique alguns princípios básicos de mecânica corporal: o dorso deve ser mantido tão retificado quanto possivel; os joelhos devem ser flexionados; a base de sustentação do corpo deve ser ampliada, por meio do posicionamento dos pés e a força deve ser feita pelos membros superiores e inferiores (11). Finocchiaro, Assaf e Finocchiaro(34) colocam que a coluna vertebral deve servir de elemento de suporte e nunca como elemento de articulação e Grandjean(35) , que as costas retas asseguram que as pressões nos discos intervertebrais sejam uniformemente distribuídas.

Segundo Knoplich (36), pressões sobre o disco intervertebral, repetitivas e freqüentes, podem ocasionar a aceleração da degeneração do mesmo, com perda de suas propriedades de amortecimento. Couto (37) destaca que o efeito do esforço físico crônico sobre a coluna vertebral é muito semelhante ao processo de envelhecimento: ocorre degeneração do disco, com diminuição do conteúdo de água do mesmo. Como conseqüência, as vértebras se tornam aproximadas $\mathrm{e}$ a coluna vertebral fica diminuída de tamanho.

Considerando-se as colocações desses autores e levando-se ainda em conta que os trabalhadores da Seção de Escolta realizam transferências durante todo o turno de trabalho, lidando com pacientes de diferentes pesos e assumindo algumas vezes posturas incorretas, sem a utilização dos princípios básicos de mecânica corporal, podemos concluir que os discos intervertebrais desses trabalhadores estão sujeitos a pressões repetitivas, que conseqüentemente podem provocar lesões sobre a coluna vertebral. Assim, se torna fundamental a elaboração de um programa de orientação e treinamento para esses trabalhadores, que aborde a utilização dos princípios de mecânica corporal, a fim de que sejam prevenidos danos sobre a coluna vertebral. 


\section{C - Dados sobre o paciente}

Entre os pacientes transferidos pelos trabalhadores da Seção de Escolta $67,9 \%$ eram do sexo masculino e $32,1 \%$ do sexo feminino.

Verificou-se que tipo de aparelhos e sondas os pacientes apresentavam no momento da transferência. $\mathrm{O}$ que se observou mais freqüentemente ( em 130 ocasiões) foi a presença de soro. Dificuldades de transferência ocorreram quando um único paciente apresentava vários aparelhos e sondas e não se contava com um número adequado de profissionais para auxiliar no procedimento. Além disso, não havia nos equipamentos utilizados um local próprio para a colocação de frascos ou recipientes, que acabavam sendo levados ou no colo do paciente (cadeira de rodas) ou sobre a maca, durante o transporte. A presença de soros, sondas, drenos de tórax e aparelhos, foram apontados em entrevistas realizadas com trabalhadores da área de saúde, em uma pesquisa desenvolvida por Alexandre e Angerami(11), como fatores que prejudicavam tanto a transferência como o transporte de pacientes pelo hospital.

A seguir procurou-se verificar o grau de dependência do paciente. Observou-se que $46 \%$ necessitaram de pequena assistência ao serem transferidos pelos trabalhadores da Seção de Escolta, sendo esses os que se deslocaram sozinhos da cama para maca ou cadeira de rodas ou vice-versa. Porém, uma porcentagem significativa de pacientes (35\%) não pode auxiliar no procedimento, exigindo um esforço físico maior por parte dos trabalhadores do setor.

Ao se planejar o levantamento de pacientes é imprescindível fazer uma avaliação completa do paciente considerando sua idade, peso, nível de consciência, capacidade de movimentação, entre outros.

Os resultados sugerem que os trabalhadores da Seção de Escolta necessitam urgentemente ter à disposição equipamentos auxiliares considerando o grande número de pacientes que dependem parcialmente ou totalmente desses trabalhadores.

Finalizando, é importante assinalar que, mover e transportar pacientes são procedimentos especializados que devem ser realizados por profissionais muito bem treinados e equipados. Sua execução demanda uma avaliação ergonômica envolvendo a análise do cliente, do método utilizado, do ambiente de trabalho e das próprias pessoas que irão executar o procedimento.

\section{CONCLUSÃo}

Os dados demonstraram que os motivos mais freqüentes de solicitação de transporte foram os encaminhamentos e retornos de exames complementares e Centro Cirúrgico, sendo que os tipos de transferências mais observadas foram da cama para cadeira de rodas e vice-versa e da cama para maca e vice-versa.

Quando o paciente era dependente e não podia cooperar, a técnica mais utilizada para realizar a transferência era com auxilio de um lençol. O número médio de pessoas que realizaram a transferência, incluindo-se os trabalhadores da Seção de Escolta e das Unidades de Internação, era de 2,3 pessoas. Foram detectadas falhas relacionadas ao planejamento principalmente envolvendo as técnicas utilizadas e o preparo do ambiente e equipamentos. Ao realizar o procedimento, os trabalhadores não utilizaram princípios de mecânica corporal, assumindo posturas inadequadas.

Observou-se também outros fatores interferindo nas transferências como pacientes obesos e que não colaboram além de um número insuficiente de pessoas participando destas. Os pacientes geralmente necessitaram de auxilio parcial ou total e estavam com soro e sondas.

Especificamente em relação aos equipamentos, observou-se que camas e macas tinham alturas bem diferentes, os equipamentos não passam por manutenção e há ausência completa de materiais e equipamentos auxiliares.

Dessa forma, os resultados do presente estudo sugerem que a existência de um setor de transporte de pacientes é uma iniciativa que deve ser estimulada. A equipe de enfermagem das unidades não precisa deslocar-se de seus setores e provavelmente está menos sujeita a lesões osteomusculares. No entanto, acreditase que os trabalhadores da Seção de Escolta necessitam urgentemente de cursos de treinamento e de terem à disposição materiais e equipamentos auxiliares.

\section{REFERÊNCIAS BIBLIOGRÁFICAS}

(1) Alexandre NMC, Angerami ELS, Moreira Filho DC. Dores nas costas e enfermagem. Rev Esc Enferm USP 1996; $30(2): 267-85$.

(2) Cato C, Olson DK, Studer M. Incidence, prevalence and variables associated with low back pain in staff nurses. AAOHN J 1989; 37(8):321-7.

(3) Marziale MHP. Condições ergonômicas da situação de trabalho, do pessoal de enfermagem, em uma unidade de internação hospitalar. [tese] Ribeirão Preto (SP): Escola de Enfermagem de Ribeirão Preto da USP;1995.

(4) Stubbs DA, Buckle PW, Hudson MP, Rivers PM, Worringham CJ. Back pain in the nursing profession I. Epidemiology and pilot methodology. Ergonomics 1983; 26(8):755-65. 
(5) Stubbs DA, Buckle PW, Hudson MP, Rivers PM. Back pain in the nursing profession II. The effectiveness of training. Ergonomics 1983; 26(8):767-79.

(6) Tate RB, Yassi A, Cooper J. Predictors of time loss after back injury in nurses. Spine 1999; 24(18): 1930-6.

(7) McAbee RR, Wilkinson NE. Back injuries registered nurses. AAOHN J 1988; 36(3): 106-12.

(8) Ryden LA. Occupational low-back injury in a hospital employee population: an epidemiologic analysis of multiple risk factors of a righ-risk occupational group. Spine 1989; 14(3):315-20.

(9) Goldman RH, Jarrard MR, Kim R, Loomis S, Atkins EH. Prioritizing back injury risk in hospital employees: application and comparison of different injury rates. J Occup Environ Med. 2000; 42(6): 645-52.

(10) Retsas A, Pinikahana J. Manual handling activities and injuries among nurses: na Australian hospital study. J Adv Nurs 2000; 31(4):875-83.

(11) Alexandre NMC, Angerami ELS. Avaliação de determinados aspectos ergonômicos no transporte de pacientes. Rev Bras Saúde Ocup 1993; 21(77):81-90.

(12) Garg A, Owen BD, Carlson B. An ergonomic evaluation of nursing assistants' job in a nursing home. Ergonomics 1992; 35(9):979-95.

(13) Owen BD, Garg, A. Reducing risk for back pain in nursing personnel. AAOHN J 1991; 39(1):24-33.

(14) Owen BD, Garg A. Back stress isn't part of the job. Amer J Nurs 1993; 93(2):48-51.

(15) Tuffnell C. Taking the strain. NZ Nurs J 1987; 80(4):10-2.

(16) Engkvist IL, Hjelm EW, Hagberg M, Menckel E, Ekenvall, L. Risk indicatiors for reported over-exertion back injuries among famale nursing personnel. Epidemiology 2000; 11(5): 519-22.

(17) Thompson C. Learning to lift. Nurs Times 1987; 83(15):34-5.

(18) Wilson M. Mind your back. Community Outlook 1986; (8):11-4.

(19) Hellsing AL, Andershed B, Bergman C, Liew M. Ergonomic education for nursing students. Int J Nurs Stud 1993; 30(6):499-510.

(20) McAtamney L, Corlett EN Ergonomic workplace assesment in a health care context. Ergonomics 1992; 35(9): 965-78.

(21) Owen BD Preventing injuries using an ergonomic approach. AORN J 2000; 72(6): 1031-6.

(22) World Health Organization. Identification and control of work-related diseases. Geneva;1985. (Techn. Rep. Ser. 714).

(23) National Back Pain Association. Royal College of nursing. The guide to the handling of_patients. London;1997.
(24) Narrow BW, Buschle KB. Fundamentals of nursing practice. New York, A Wiley Medical Publication;1982.

(25) Tuohy-Main K. A manual of handling people: a health and safety guide for carers. Adelaide: Helios Art and Book $\mathrm{CO} ; 1994$.

(26) Unison Lifting and back pain in healthcare work: guidance for health care workers. London; 1992.

(27) Bell F. Ergonomic aspects of equipament. Int J Nurs Stud 1987; 24(4):331-7.

(28) Professional development. Lifting and handling. Knowledge for practice. Nurs Times 1995;91(1).

(29) Back care. Preventing injury. Nurs Times 1995; 91(43): 44-6.

(30) Seymour J. Handling Aids. Lifting and moving patients. Nurs Times 1995; 91(27): 48-50.

(31) Harber P, Pena L, Hsu P, Billet E, Greer D, Kim K. Personal history, training, and worksite as predictors of back pain of nurses. Am J Ind Med 1994;25(4):519-26.

(32) Larcombe J. Manual handling. Too heavy to handle? Nurs Times. 1993; 89(40):46,48,50.

(33) Owen BD, Fragala G. Reducing perceived physical stress while transferring residents. An ergonomic approach. AAOHN J 1999; 47(7):316-23.

(34) Finocchiaro J, Assaf DL, Finocchiaro M. Manual de prevenção das lombalgias. São Paulo: Lex;1978.

(35) Grandjean E. Lifting the task to the man. 4.ed. London: Taylor \& Francis; 1988 .

(36) Knoplich J. Enfermidades da coluna vertebral 2.ed. São Paulo: Panamed; 1986.

(37) Couto HA. Temas de saúde ocupacional. Belo Horizonte: Ergo; 1987. 